\title{
Compact Weighted Composition Operators and Multiplication Operators between Hardy Spaces
}

\author{
Sei-Ichiro Ueki ${ }^{1}$ and Luo Luo ${ }^{2}$ \\ ${ }^{1}$ Department of Mathematics, Faculty of Science Division II, Tokyo University of Science, \\ 4-6-1 Higashicho, Hitachi, Ibaraki 317-0061, Japan \\ ${ }^{2}$ Department of Mathematics, University of Science and Technology of China, Hefei 230026, China
}

Correspondence should be addressed to Sei-Ichiro Ueki, sueki@camel.plala.or.jp

Received 27 August 2007; Accepted 10 February 2008

Recommended by Stephen Clark

We estimate the essential norm of a compact weighted composition operator $u C_{\varphi}$ acting between different Hardy spaces of the unit ball in $\mathbb{C}^{N}$. Also we will discuss a compact multiplication operator between Hardy spaces.

Copyright ( 92008 S.-I. Ueki and L. Luo. This is an open access article distributed under the Creative Commons Attribution License, which permits unrestricted use, distribution, and reproduction in any medium, provided the original work is properly cited.

\section{Introduction}

Let $N$ be a fixed integer. Let $B_{N}$ denote the unit ball of $\mathbb{C}^{N}$ and let $H\left(B_{N}\right)$ denote the space of all holomorphic functions in $B_{N}$. For each $p, 1 \leq p<\infty$, the Hardy space $H^{p}\left(B_{N}\right)$ is defined by

$$
\begin{gathered}
H^{p}\left(B_{N}\right)=\left\{f \in H\left(B_{N}\right): \sup _{0<r<1} \int_{\partial B_{N}}|f(r \zeta)|^{p} d \sigma(\zeta)<\infty\right\}, \\
\|f\|_{p}=\left[\int_{\partial B_{N}}\left|f^{*}(\zeta)\right|^{p} d \sigma(\zeta)\right]^{1 / p},
\end{gathered}
$$

where $d \sigma$ is the normalized Lebesgue measure on the boundary $\partial B_{N}$ of $B_{N}$.

For a given holomorphic self-map $\varphi$ of $B_{N}$ and holomorphic function $u$ in $B_{N}$, the weighted composition operator $u C_{\varphi}$ is defined by $u C_{\varphi} f=u(f \circ \varphi)$. In particular, if $u$ is the constant function 1 , then $u C_{\varphi}$ becomes the composition operator $C_{\varphi}$. In the special case that $\varphi$ is the identity mapping of $B_{N}, u C_{\varphi}$ is called the multiplication operator and is denoted by $M_{u}$.

Let $X$ and $Y$ be Banach spaces. For a bounded linear operator $T: X \rightarrow Y$, the essential norm $\|T\|_{e, X \rightarrow Y}$ is defined to be the distance from $T$ to the set of the compact operators $\mathcal{K}$, namely,

$$
\|T\|_{e, X \rightarrow Y}=\inf \{\|T-\mathcal{K}\|: \mathcal{K} \text { is compact from } X \text { into } Y\},
$$


where $\|\cdot\|$ denotes the usual operator norm. Clearly, $T$ is compact if and only if $\|T\|_{e, X \rightarrow Y}=0$. Thus, the essential norm is closely related to the compactness problem of concrete operators. Many mathematicians have studied the essential norm of various concrete operators. For these studies about composition operators on Hardy spaces of the unit disc, refer to [1-4]. In this paper, our objects are weighted composition operators between Hardy spaces of the unit ball $B_{N}$. Several authors have also studied weighted composition operators on various analytic function spaces. For more information about weighted composition operators, refer to [5-10].

Recently, Contreras and Hernández-Díaz [11,12] have characterized the compactness of $u C_{\varphi}$ from $H^{p}\left(B_{1}\right)$ into $H^{q}\left(B_{1}\right)(1<p \leq q<\infty)$ in terms of the pull-back measure. Here, $B_{1}$ denotes the open unit disc in the complex plane. But they have not given the estimate for the essential norm of $u C_{\varphi}$. The essential norm of $u C_{\varphi}: H^{p}\left(B_{1}\right) \rightarrow H^{q}\left(B_{1}\right)$ has been studied by Čučković and Zhao [13, 14]. In the higher-dimensional case, Ueki [15] characterized the boundedness and compactness of $u C_{\varphi}: H^{p}\left(B_{N}\right) \rightarrow H^{q}\left(B_{N}\right)$, in terms of the pull-back measure and the integral operator. The purpose of this paper is to estimate the essential norm of $u C_{\varphi}$ : $H^{p}\left(B_{N}\right) \rightarrow H^{q}\left(B_{N}\right)$. The following theorem is our main result.

Main Theorem. Let $1<p \leq q<\infty$. If $u C_{\varphi}$ is a bounded weighted composition operator from $H^{p}\left(B_{N}\right)$ into $H^{q}\left(B_{N}\right)$, then

$$
\begin{aligned}
\left\|u C_{\varphi}\right\|_{e, H^{p} \rightarrow H^{q}}^{q} & \sim \limsup _{|w| \rightarrow 1^{-}} \int_{\partial B_{N}}\left|u^{*}(\zeta)\right|^{q}\left\{\frac{1-|w|^{2}}{\left|1-\left\langle\varphi^{*}(\zeta), w\right\rangle\right|^{2}}\right\}^{q N / p} d \sigma(\zeta) \\
& \sim \limsup \sup _{t \rightarrow 0^{+}} \frac{\mu_{\zeta, u}(S(\zeta, t))}{t^{q N / p}}
\end{aligned}
$$

where $\mu_{\varphi, u}$ is the pull-back measure induced by $\varphi$ and $u, S(\zeta, t)$ is the Carleson set of $\overline{B_{N}}$, and the notation $\sim$ means that the ratios of two terms are bounded below and above by constants dependent upon the dimension $N$ and other parameters.

The one variable case of the first estimate for $\left\|u C_{\varphi}\right\|_{e}$ in above theorem may be found in the work [14] by Čučković and Zhao. In the case $p=q=2$ and $u=1$, Choe [1] and Luo [16] showed that the essential norm $\left\|C_{\varphi}\right\|_{e}$ is comparable to the value $\lim \sup _{t \rightarrow 0^{+}} \sup _{\zeta \in \partial B_{N}}\left(\mu_{\varphi}(S(\zeta, t)) / t^{N}\right)$.

We give the proof of main theorem in Section 3. The ideas of our proofs are based on the method which Choe or Luo used in their papers. In Section 4, we have a discussion on the compact multiplication operator between different Hardy spaces.

Throughout the paper, the symbol $C$ denotes a positive constant, possibly different at each occurrence, but always independent of the function $f$ and other parameters $r$ or $t$.

\section{Carleson-type measures}

For each $u \in H^{q}\left(B_{N}\right)$, we can define a finite positive Borel measure $\mu_{\varphi, u}$ on $\overline{B_{N}}$ by

$$
\mu_{\varphi, u}(E)=\int_{\varphi^{*-1}(E)}\left|u^{*}\right|^{q} d \sigma \quad\left(\forall \text { Borel sets } E \text { of } \overline{B_{N}}\right),
$$


where $\varphi^{*}$ denotes the radial limit map of the mapping $\varphi$ considered as a map of $\partial B_{N} \rightarrow \overline{B_{N}}$. A change-of-variable formula from measure theory shows that

$$
\int_{\overline{B_{N}}} g d \mu_{\varphi, u}=\int_{\partial B_{N}}\left|u^{*}\right|^{q}\left(g \circ \varphi^{*}\right) d \sigma
$$

for each nonnegative measurable function $g$ on $\overline{B_{N}}$. This type of pull-back measure played an important role in past studies of composition operators on Hardy spaces of $B_{N}$.

For each $\zeta \in \partial B_{N}$ and $t>0$, let

$$
\begin{gathered}
S(\zeta, t)=\left\{z \in \overline{B_{N}}:|1-\langle z, \zeta\rangle|<t\right\}, \\
B(\zeta, t)=S(\zeta, t) \cap B_{N}, \quad Q(\zeta, t)=S(\zeta, t) \cap \partial B_{N} .
\end{gathered}
$$

It is well known that $\sigma(Q(\zeta, t))$ is comparable to $t^{N}$ ([17, page 67]).

The proof of the following lemma is essentially the same as that of Power's theorem in [18].

Lemma 2.1. Let $1 \leq \alpha<\infty$. Suppose that $\mu$ is a positive Borel measure on $B_{N}$ and that there exists a constant $C>0$ such that

$$
\mu(B(\zeta, t)) \leq C t^{\alpha N} \quad\left(\zeta \in \partial B_{N}, t>0\right) .
$$

Then there exists a constant $K>0$ such that

$$
\left[\int_{B_{N}}|f|^{p \alpha} d \mu\right]^{1 / p \alpha} \leq K\|f\|_{H^{p}} \quad\left(f \in H^{p}\left(B_{N}\right)\right) .
$$

Proof. Fix $f \in H^{p}\left(B_{N}\right)$ and $s>0$. By the same argument as in the proof of theorem in [18, pages 14-15], it follows from (2.4) that there exists a constant $C>0$ such that

$$
\mu\left(\left\{z \in B_{N}:|f(z)| \geq s\right\}\right) \leq C\left[\sigma\left(\left\{\zeta \in \partial B_{N}: M f(\zeta) \geq s\right\}\right)\right]^{\alpha},
$$

where $M f$ is the admissible maximal function of $f$ which is defined by

$$
M f(\zeta)=\sup \left\{|f(z)|: z \in \mathbb{C}^{n},|1-\langle z, \zeta\rangle|<1-|z|^{2}\right\},
$$

for $\zeta \in \partial B_{N}$. By (2.6), we have

$$
\int_{B_{N}}|f|^{p \alpha} d \mu=p \alpha \int_{0}^{\infty} \mu\{|f|>s\} s^{p \alpha-1} d s \leq C p \alpha \int_{0}^{\infty} \sigma\{M f \geq s\}^{\alpha} s^{p \alpha-1} d s .
$$

Since $f \in H^{p}\left(B_{N}\right)$, it follows from [17, Theorem 5.6.5] that

$$
\sigma\{M f \geq s\}^{\alpha-1} s^{p \alpha-p} \leq\left[\int_{\partial B_{N}}\{M f(\zeta)\}^{p} d \sigma(\zeta)\right]^{\alpha-1} \leq C\|f\|_{H^{p}}^{p(\alpha-1)} .
$$

By (2.8) and (2.9), we have

$$
\begin{aligned}
\int_{B_{N}}|f|^{p \alpha} d \mu & \leq C\|f\|_{H^{p}}^{p(\alpha-1)} p \int_{0}^{\infty} \sigma\{M f \geq s\} s^{p-1} d s \\
& \leq C\|f\|_{H^{p}}^{p(\alpha-1)} \int_{\partial B_{N}}\{M f(\zeta)\}^{p} d \sigma(\zeta) \leq C\|f\|_{H^{p}}^{p \alpha} .
\end{aligned}
$$

This completes the proof. 
Lemma 2.2. Let $1 \leq \alpha<\infty$. Suppose that $\mu$ is a positive Borel measure on $\partial B_{N}$ such that

$$
\mu(Q(\zeta, t)) \leq C t^{\alpha N} \quad\left(\zeta \in \partial B_{N}, t>0\right)
$$

for some constant $C>0$.

(a) If $\alpha=1$, then there exist a $g \in L^{\infty}\left(\partial B_{N}\right)$ and a constant $C^{\prime}>0$ ( $C^{\prime}$ is the product of $C$ and $a$ constant depending only on the dimension $N$ ) such that $d \mu=g d \sigma$ and $\|g\|_{L^{\infty}} \leq C^{\prime}$.

(b) If $\alpha>1$, then $\mu \equiv 0$ for all Borel sets of $\partial B_{N}$.

Proof. Part (a) is completely analogous to [19, page 238, Lemma 1.3]. So we only prove part (b). Combining $\sigma(Q(\zeta, t)) \sim t^{N}$ with $(2.11)$, we have

$$
\frac{\mu(Q(\zeta, t))}{\sigma(Q(\zeta, t))} \leq C t^{N(\alpha-1)}
$$

for all $\zeta \in \partial B_{N}$ and $t>0$. Hence we see that the maximal function $M \mu$ of the positive measure $\mu$ satisfies $M \mu(\zeta)<\infty$ for all $\zeta \in \partial B_{N}$. By [17, page 70, Theorem 5.2.7], we obtain $d \mu=g d \sigma$ for some $g \in L^{1}\left(\partial B_{N}\right)$. By (2.12), we have

$$
0 \leq \frac{1}{\sigma(Q(\zeta, t))} \int_{Q(\zeta, t)} g d \sigma=\frac{\mu(Q(\zeta, t))}{\sigma(Q(\zeta, t))} \leq C t^{N(\alpha-1)}
$$

for all $\zeta \in \partial B_{N}$ and $t>0$. Letting $t \rightarrow 0^{+}$, we see that $g=0$ a.e. on $\partial B_{N}$, and so $\mu \equiv 0$. This completes the proof of part (b).

Combining Lemma 2.1 with Lemma 2.2 and using the same argument as in [19, page 239], we obtain the following lemma.

Lemma 2.3. Let $1<p \leq q<\infty$. Suppose that $\mu$ is a positive Borel measure on $\overline{B_{N}}$ such that

$$
\mu(S(\zeta, t)) \leq C t^{q N / p} \quad\left(\zeta \in \partial B_{N}, t>0\right),
$$

for some constant $C>0$. Then, there exists a constant $K>0$ such that

$$
\left[\int_{\overline{B_{N}}}\left|f^{*}\right|^{q} d \mu\right]^{1 / q \leq K\|f\|_{H^{p}}}
$$

for all $f \in H^{p}\left(B_{N}\right)$. Here, the notation $f^{*}$ denotes the function defined on $\overline{B_{N}}$ by $f^{*}=f$ in $B_{N}$ and $f^{*}=\lim _{r \rightarrow 1^{-}} f_{r}$ a.e. $[\sigma]$ on $\partial B_{N}$.

Remark 2.4. In Lemma 2.3 (or in Lemma 2.1), we see that the constant $K$ of (2.15) (or (2.5)) can be chosen to be the product of $C$ and a positive constant depending only on $p, q$, and the dimension $N$.

In order to prove the main theorem, we need some results. These are the extensions of [19, Corollary 1.4 and Lemma 1.6] to the case of weighted composition operators $u C_{\varphi}$. 
Proposition 2.5. Let $1<p \leq q<\infty$. Suppose that $\varphi: B_{N} \rightarrow B_{N}$ is a holomorphic map and $u \in H^{q}\left(B_{N}\right) \backslash\{0\}$ such that $u C_{\varphi}: H^{p}\left(B_{N}\right) \rightarrow H^{q}\left(B_{N}\right)$ is bounded. Then $\varphi^{*}$ cannot carry a set of positive $\sigma$-measure in $\partial B_{N}$ into a set of $\sigma$-measure 0 in $\partial B_{N}$.

Proof. Suppose that $E, F \subset \partial B_{N}$ and $\varphi^{*}(E) \subset F$ with $\sigma(E)>0$ and $\sigma(F)=0$. Put $\mu=\left.\mu_{\varphi, u}\right|_{\partial B_{N}}$. As in the case of composition operators, it is well known that the boundedness of $u C_{\varphi}: H^{p}\left(B_{N}\right) \rightarrow$ $H^{q}\left(B_{N}\right)$ implies

$$
\mu(S(\zeta, t)) \leq C t^{q N / p} \quad\left(\zeta \in \partial B_{N}, t>0\right),
$$

for some positive constant $C$ (see [15]). By Lemma 2.2, we see that $\mu \equiv 0$ (if $p<q$ ) or $\mu$ is absolutely continuous with respect to $d \sigma$ (if $p=q$ ). Thus we have

$$
0 \geq \mu\left(\varphi^{*}(E)\right) \equiv \int_{\varphi^{*-1}\left(\varphi^{*}(E)\right)}\left|u^{*}\right|^{q} d \sigma \geq \int_{E}\left|u^{*}\right|^{q} d \sigma
$$

That is, $u^{*}=0$ a.e. on $E$. Hence [17, page 83, Theorem 5.5.9] gives that $u \equiv 0$ in $B_{N}$. This contradicts $u \neq 0$.

Lemma 2.6. Let $1<p \leq q<\infty$ and $f \in H^{p}\left(B_{N}\right)$. Suppose that $\varphi: B_{N} \rightarrow B_{N}$ is a holomorphic map and $u \in H^{q}\left(B_{N}\right) \backslash\{0\}$ such that $u C_{\varphi}: H^{p}\left(B_{N}\right) \rightarrow H^{q}\left(B_{N}\right)$ is bounded. Then $u^{*}(f \circ \varphi)^{*}=u^{*}\left(f^{*} \circ \varphi^{*}\right)$ a.e. $[\sigma]$ on $\partial B_{N}$. Here the notation $f^{*}$ is used as in Lemma 2.3.

Proof (cf. [19, Lemma 1.6]). Since $\varphi^{*}$ cannot carry a set of positive measure in $\partial B_{N}$ into a set of measure 0 in $\partial B_{N}$ (by Proposition 2.5) and since the radial limit of $\varphi, f$ and $\psi$ exist on a set of full measure in $\partial B_{N}$, we have $\lim _{r \rightarrow 1^{-}} u^{*}\left(f_{r} \circ \varphi^{*}\right)=u^{*}\left(f^{*} \circ \varphi^{*}\right)$ a.e. $[\sigma]$ on $\partial B_{N}$.

On the other hand, since $f_{r}$ is in the ball algebra $A\left(B_{N}\right)$ and $f_{r} \rightarrow f$ as $r \rightarrow 1^{-}$in $H^{p}\left(B_{N}\right)$, the boundedness of $u C_{\varphi}$ shows that

$$
\begin{aligned}
0 & \leq \int_{\partial B_{N}}\left|u^{*}(\zeta)(f \circ \varphi)^{*}(\zeta)-u^{*}(\zeta)\left(f^{*} \circ \varphi^{*}\right)(\zeta)\right|^{q} d \sigma(\zeta) \\
& \leq \liminf _{r \rightarrow 1^{-}} \int_{\partial B_{N}}\left|u^{*}(\zeta)(f \circ \varphi)^{*}(\zeta)-u^{*}(\zeta)\left(f_{r} \circ \varphi\right)^{*}(\zeta)\right|^{q} d \sigma(\zeta) \\
& =\liminf _{r \rightarrow 1^{-}}\left\|u C_{\varphi} f-u C_{\varphi} f_{r}\right\|_{H^{q}}^{q}=0 .
\end{aligned}
$$

This implies that $u^{*}(f \circ \varphi)^{*}=u^{*}\left(f^{*} \circ \varphi^{*}\right)$ a.e. $[\sigma]$ on $\partial B_{N}$.

\section{Weighted composition operators between Hardy spaces}

Theorem 3.1. Let $1<p \leq q<\infty$. If $u C_{\varphi}$ is a bounded weighted composition operator from $H^{p}\left(B_{N}\right)$ into $H^{q}\left(B_{N}\right)$, then

$$
\begin{aligned}
\left\|u C_{\varphi}\right\|_{e, H^{p} \rightarrow H^{q}}^{q} & \sim \limsup _{|w| \rightarrow 1^{-}} \int_{\partial B_{N}}\left|u^{*}(\zeta)\right|^{q}\left\{\frac{1-|w|^{2}}{\left|1-\left\langle\varphi^{*}(\zeta), w\right\rangle\right|^{2}}\right\}^{q N / p} d \sigma(\zeta) \\
& \sim \limsup \sup _{t \rightarrow 0^{+}} \frac{\mu_{\varphi, u}(S(\zeta, t))}{t^{q N / p}}
\end{aligned}
$$


Proof of the lower estimates. For each $w \in B_{N}$, we define the function $f_{w}$ on $\overline{B_{N}}$ by

$$
f_{w}(z)=\left\{\frac{1-|w|^{2}}{(1-\langle z, w\rangle)^{2}}\right\}^{N / p}
$$

Then the functions $\left\{f_{w}: w \in B_{N}\right\}$ belong to the ball algebra $A\left(B_{N}\right)$ and form a bounded sequence of $H^{p}\left(B_{N}\right)$. Take a compact operator $\mathcal{K}: H^{p}\left(B_{N}\right) \rightarrow H^{q}\left(B_{N}\right)$ arbitrarily. Since the bounded sequence $\left\{f_{w}\right\}$ converges to 0 uniformly on compact subsets of $B_{N}$ as $|w| \rightarrow 1^{-}$, we have $\left\|\mathcal{K} f_{w}\right\|_{H^{q}} \rightarrow 0$ as $|w| \rightarrow 1^{-}$. Thus we obtain

$$
\left\|u C_{\varphi}-\mathcal{K}\right\|_{H^{p} \rightarrow H^{q}} \geq C \limsup _{|w| \rightarrow 1^{-}}\left\|\left(u C_{\varphi}-\mathcal{K}\right) f_{w}\right\|_{H^{q}} \geq C \underset{|w| \rightarrow 1^{-}}{\limsup }\left\|u C_{\varphi} f_{w}\right\|_{H^{q}} .
$$

By the definition of $f_{w}$, we also see that

$$
\left\|u C_{\varphi} f_{w}\right\|_{H^{q}}^{q}=\int_{\partial B_{N}}\left|u^{*}(\zeta)\right|^{q}\left\{\frac{1-|w|^{2}}{\left|1-\left\langle\varphi^{*}(\zeta), w\right\rangle\right|^{2}}\right\}^{q N / p} d \sigma(\zeta) .
$$

Combining this with (3.3), we get

$$
\left\|u C_{\varphi}-\mathcal{K}\right\|_{H^{p} \rightarrow H^{q}}^{q} \geq C \limsup _{|w| \rightarrow 1^{-}} \int_{\partial B_{N}}\left|u^{*}(\zeta)\right|^{q}\left\{\frac{1-|w|^{2}}{\left|1-\left\langle\varphi^{*}(\zeta), w\right\rangle\right|^{2}}\right\}^{q N / p} d \sigma(\zeta) .
$$

Since this holds for every compact operator $\mathcal{K}$, it follows that

$$
\left\|u C_{\varphi}\right\|_{e, H^{p} \rightarrow H^{q}}^{q} \geq C \limsup _{|w| \rightarrow 1^{-}} \int_{\partial B_{N}}\left|u^{*}(\zeta)\right|^{q}\left\{\frac{1-|w|^{2}}{\left|1-\left\langle\varphi^{*}(\zeta), w\right\rangle\right|^{2}}\right\}^{q N / p} d \sigma(\zeta) .
$$

Furthermore, we put $w=(1-t) \zeta$ for each $t, 0<t<1$ and $\zeta \in \partial B_{N}$ in the definition of $f_{w}$. Since we see that $\left|f_{(1-t) \zeta}(z)\right| \geq C t^{-q N / p}$ for all $z \in S(\zeta, t)$, we have

$$
C \sup _{\zeta \in \partial B_{N}} \frac{\mu_{\varphi, u}(S(\zeta, t))}{t^{q N / p}} \leq \sup _{\zeta \in \partial B_{N}} \int_{S(\zeta, t)}\left|f_{(1-t) \zeta}\right|^{q} d \mu_{\varphi, u} \leq \sup _{\zeta \in \partial B_{N}}\left\|u C_{\varphi} f_{(1-t) \zeta}\right\|_{H^{q}}^{q} .
$$

Letting $t \rightarrow 0^{+}$, we get

$$
C \limsup \sup _{t \rightarrow 0^{+}} \frac{\mu_{\varphi, u}(S(\zeta, t))}{t^{q N / p}} \leq \lim \sup _{t \rightarrow 0^{+}} \sup _{\zeta \in \partial B_{N}}\left\|u C_{\varphi} f_{(1-t) \zeta}\right\|_{H^{q}}^{q} .
$$

Combining this with (3.6), we obtain

$$
C \limsup \sup _{t \rightarrow 0^{+}} \frac{\mu_{\varphi, u}(S(\zeta, t))}{t^{q N / p}} \leq\left\|u C_{\varphi}\right\|_{e, H^{p} \rightarrow H^{q}}^{q},
$$

completing the proof of the lower estimates. 
To prove the upper estimates, we need some technical results about the polynomial approximation of $f \in H^{p}\left(B_{N}\right)$. Recall that a holomorphic function $f$ in $B_{N}$ has the homogeneous expansion

$$
f(z)=\sum_{k=0}^{\infty} \sum_{|\gamma|=k} c(\gamma) z^{\gamma},
$$

where $\gamma=\left(\gamma_{1}, \ldots, \gamma_{N}\right)$ is a multi-index, $|\gamma|=\gamma_{1}+\cdots+\gamma_{N}$, and $z^{\gamma}=z_{1} \gamma_{1} \cdots z_{N}^{\gamma_{N}}$. For the homogeneous expansion of $f$ and any integer $n \geq 1$, let

$$
R_{n} f(z)=\sum_{k=n}^{\infty} \sum_{|\gamma|=k} c(\gamma) z^{\gamma}
$$

and $K_{n}=I-R_{n}$, where $I f=f$ is the identity operator.

Proposition 3.2. Suppose that $X$ is a Banach space of holomorphic functions in $B_{N}$ with the property that the polynomials are dense in $X$. Then $\left\|K_{n} f-f\right\|_{X} \rightarrow 0$ as $n \rightarrow \infty$ if and only if $\sup \left\{\left\|K_{n}\right\|: n \geq\right.$ $1\}<\infty$.

Proof. We see that [20, Proposition 1] also holds if we replace the unit disc with the unit ball. So we omit the proof of this proposition.

Proposition 3.3. If $1<p<\infty$, then $\left\|K_{n} f-f\right\|_{H^{p}} \rightarrow 0$ as $n \rightarrow \infty$ for each $f \in H^{p}\left(B_{N}\right)$.

Proof. For each $f \in H^{p}\left(B_{N}\right)$ and $r, 0<r<1$, the slice function $\left(f_{r}\right)_{\zeta}\left(\zeta \in \partial B_{N}\right)$ of $f_{r}$ is in the disc algebra $A(\mathbb{D})$. Here, $f_{r}$ denotes the dilated function of $f$, that is $f_{r}(z)=f(r z)$. Hence [20, Corollary 3 and Proposition 1] implies that there is a positive constant $C_{p}$ such that

$$
\frac{1}{2 \pi} \int_{-\pi}^{\pi}\left|K_{n}\left(f_{r}\right)_{\zeta}\left(e^{i \theta}\right)\right|^{p} d \theta \leq C_{p} \frac{1}{2 \pi} \int_{-\pi}^{\pi}\left|\left(f_{r}\right)_{\zeta}\left(e^{i \theta}\right)\right|^{p} d \theta,
$$

for every integer $n \geq 1$. Since $K_{n}\left(f_{r}\right)_{\zeta}\left(e^{i \theta}\right)=K_{n} f\left(r e^{i \theta} \zeta\right)$, integration by slices (see [17, page 15, Proposition 1.4.7.]) shows

$$
\int_{\partial B_{N}}\left|K_{n} f(r \zeta)\right|^{p} d \sigma(\zeta) \leq C_{p} \int_{\partial B_{N}}|f(r \zeta)|^{p} d \sigma(\zeta)
$$

that is, $\left\|K_{n}\right\| \leq C_{p}^{1 / p}$ for every integer $n \geq 1$. By Proposition 3.2, we see $\left\|K_{n} f-f\right\|_{H^{p}} \rightarrow 0$ as $n \rightarrow \infty$. This completes the proof of the proposition.

Corollary 3.4. If $1<p<\infty$, then $R_{n}$ converges to 0 pointwise in $H^{p}\left(B_{N}\right)$ as $n \rightarrow \infty$. Moreover, $\sup \left\{\left\|R_{n}\right\|: n \geq 1\right\}<\infty$.

Proof. Since $R_{n} f=f-K_{n} f$, Proposition 3.3 shows that $\left\|R_{n} f\right\|_{p} \rightarrow 0$ as $n \rightarrow \infty$. Furthermore, the principle of uniform boundedness implies that $\sup _{n \geq 1}\left\|R_{n}\right\|<\infty$.

Lemma 3.5. Let $1<p<\infty$. For each $f \in H^{p}\left(B_{N}\right)$ and $n \geq 1$,

$$
\left|R_{n} f(z)\right| \leq\|f\|_{H^{p}} \sum_{k=n}^{\infty} \frac{\Gamma(N+k)}{k ! \Gamma(N)}|z|^{k} .
$$


Proof. Let $K_{w}$ be the reproducing kernel for $H^{2}\left(B_{N}\right)$ and let $C[f]$ be the Cauchy-Szegö projection. Then, the orthogonality of monomials $\zeta^{\alpha}$ implies that

$$
R_{n} f(z)=C\left[R_{n} f\right](z)=\int_{\partial B_{N}} R_{n} f(\zeta) \overline{K_{z}(\zeta)} d \sigma(\zeta)=\int_{\partial B_{N}} f(\zeta) \overline{R_{n} K_{z}(\zeta)} d \sigma(\zeta)
$$

Hölder's inequality and the expansion of $K_{z}(w)$ give

$$
\begin{aligned}
\left|R_{n} f(z)\right| & \leq \int_{\partial B_{N}}|f(\zeta)|\left|R_{n} K_{z}(\zeta)\right| d \sigma(\zeta) \\
& \leq\left\{\int_{\partial B_{N}}|f(\zeta)|^{p} d \sigma(\zeta)\right\}^{1 / p}\left\{\int_{\partial B_{N}}\left|R_{n} K_{z}(\zeta)\right|^{q} d \sigma(\zeta)\right\}^{1 / q} \\
& \leq\|f\|_{H^{p}} \sum_{k=n}^{\infty} \frac{\Gamma(N+k)}{k ! \Gamma(N)}|z|^{k} .
\end{aligned}
$$

This completes the proof.

The following lemma is well known in the case of functional Hilbert spaces (cf. $[4,21]$ ). As in the proof of [21, Lemma 3.16], an elementary argument verifies Lemma 3.6.

Lemma 3.6. Let $1<p \leq q<\infty$. If $u C_{\varphi}$ is bounded from $H^{p}\left(B_{N}\right)$ into $H^{q}\left(B_{N}\right)$, then

$$
\left\|u C_{\varphi}\right\|_{e, H^{p} \rightarrow H^{q}} \leq \liminf _{n \rightarrow \infty}\left\|u C_{\varphi} R_{n}\right\|_{H^{p} \rightarrow H^{q}} .
$$

Let us prove the upper estimates for the essential norm of $u C_{\varphi}$.

Proof of the upper estimates. For the sake of convenience, we set

$$
\begin{gathered}
M_{1}=\limsup _{|w| \rightarrow 1^{-}} \int_{\partial B_{N}}\left|u^{*}(\zeta)\right|^{q}\left\{\frac{1-|w|^{2}}{\left|1-\left\langle\varphi^{*}(\zeta), w\right\rangle\right|^{2}}\right\}^{(q N / p)} d \sigma(\zeta), \\
M_{2}=\limsup _{t \rightarrow 0^{+}} \sup _{\zeta \in \partial B_{N}} \frac{\mu_{\varphi, u}(S(\zeta, t))}{t^{q N / p}}, \\
D(\zeta, t)=\left\{z \in \overline{B_{N}}:|z|>1-t, \frac{z}{|z|} \in Q(\zeta, t)\right\} .
\end{gathered}
$$

By the notation (3.18), for given $\varepsilon>0$, we can choose an $R_{1}, 0<R_{1}<1$ such that

$$
\int_{\partial B_{N}}\left|u^{*}(\zeta)\right|^{q}\left\{\frac{1-|w|^{2}}{\left|1-\left\langle\varphi^{*}(\zeta), w\right\rangle\right|^{2}}\right\}^{q N / p} d \sigma(\zeta)<M_{1}+\varepsilon,
$$

for $w \in B_{N}$ with $|w| \geq R_{1}$. For each $\zeta \in \partial B_{N}$ and $t, 0<t \leq 1-R_{1} \equiv t_{1}$, we put $w_{1}=(1-t) \zeta$. Since the function $f_{w_{1}}(z)=\left\{\left(1-\left|w_{1}\right|^{2}\right) /\left(1-\left\langle z, w_{1}\right\rangle\right)^{2}\right\}^{N / p}$ satisfies $\left|f_{w_{1}}(z)\right|^{p}>4^{-N} t^{-N}$ for all 
$z \in S(\zeta, t)$, the inequality (3.21) implies that

$$
\frac{\mu_{\varphi, u}(S(\zeta, t))}{t^{q N / p}}<C \int_{S(\zeta, t)}\left|f_{w_{1}}(z)\right|^{q} d \mu_{\varphi, u}(z)<C\left(M_{1}+\varepsilon\right)
$$

for all $\zeta \in \partial B_{N}$ and all $t, 0<t \leq t_{1}$.

By the notation (3.19), we can also choose a $t_{2}, 0<t_{2}<1$, so that

$$
\sup _{\zeta \in \partial B_{N}} \frac{\mu_{\varphi, u}(S(\zeta, t))}{t^{q N / p}}<M_{2}+\varepsilon
$$

for all $t, 0<t \leq t_{2}$. Let $\mu_{1}$ and $\mu_{2}$ be the restrictions of $\mu_{\varphi, u}$ to $\overline{B_{N}} \backslash\left(1-t_{1}\right) \overline{B_{N}}$ and $\overline{B_{N}} \backslash\left(1-t_{2}\right) \overline{B_{N}}$, respectively. We claim that $\mu_{j}(j=1,2)$ also satisfies the Carleson measure condition

$$
\mu_{j}(S(\zeta, t)) \leq C\left(M_{j}+\varepsilon\right) t^{q N / p}
$$

for all $\zeta \in \partial B_{N}$ and $t>0$. By (3.22) or (3.23), these conditions are true for all $t, 0<t \leq t_{j}$. Hence, we assume that $t>t_{j}$. For a finite cover $\left\{Q\left(w_{k}, t_{j} / 3\right)\right\}$, where $w_{k} \in Q(\zeta, t)$ of the set $\bar{Q}(\zeta, t)=\left\{z \in \partial B_{N}:|1-\langle z, \zeta\rangle| \leq t\right\}$, the covering property implies that there exists a disjoint subcollection $\Gamma$ of $\left\{Q\left(w_{k}, t_{j} / 3\right)\right\}$ so that

$$
Q(\zeta, t) \subset \bigcup_{\Gamma} Q\left(w_{k}, t_{j}\right)
$$

Furthermore, we obtain $\operatorname{card}(\Gamma) \leq C\left(t / t_{j}\right)^{N}$. By the notation (3.20), we have

$$
\begin{aligned}
\mu_{j}(S(\zeta, t)) & \leq \mu_{j}(D(\zeta, t)) \leq \sum_{\Gamma} \mu_{j}\left(D\left(w_{k}, t_{j}\right)\right) \\
& \leq \sum_{\Gamma} \mu_{j}\left(S\left(w_{k}, 2 t_{j}\right)\right) \leq C\left(\frac{t}{t_{j}}\right)^{N}\left(M_{j}+\varepsilon\right) t_{j}{ }^{q N / p} \\
& =C\left(M_{j}+\varepsilon\right) t^{N} t_{j}{ }^{(q / p-1) N} \leq C\left(M_{j}+\varepsilon\right) t^{q N / p},
\end{aligned}
$$

where the constant $C$ depends only on $p, q$, and the dimension $N$.

Now, we take a function $f \in H^{p}\left(B_{N}\right)$ with $\|f\|_{H^{p}} \leq 1$. By Lemma 2.6, we have

$$
\begin{aligned}
\left\|u C_{\varphi} R_{n} f\right\|_{H^{q}}^{q} & =\int_{\partial B_{N}}\left|u^{*}\left(R_{n} f^{*} \circ \varphi^{*}\right)\right|^{q} d \sigma \\
& =\int_{\overline{B_{N}}}\left|R_{n} f^{*}\right|^{q} d \mu_{\varphi, u} \\
& =\int_{\overline{B_{N}}}\left|R_{n} f^{*}\right|^{q} d \mu_{j}+\int_{\left(1-t_{j}\right) \overline{B_{N}}}\left|R_{n} f\right|^{q} d \mu_{\varphi, u}
\end{aligned}
$$

for all integers $n \geq 1$. Condition (3.24) and Lemma 2.3 implies that

$$
\int_{\overline{B_{N}}}\left|R_{n} f^{*}\right|^{q} d \mu_{j} \leq C\left(M_{j}+\varepsilon\right)\left\|R_{n} f\right\|_{H^{p}}^{q} \leq C \sup _{n \geq 1}\left\|R_{n}\right\|^{q}\left(M_{j}+\varepsilon\right) .
$$


On the other hand, by Lemma 3.5, we have

$$
\int_{\left(1-t_{j}\right) \overline{B_{N}}}\left|R_{n} f\right|^{q} d \mu_{\varphi, u} \leq\|f\|_{H^{p}}^{q}\left\{\sum_{k=n}^{\infty} \frac{\Gamma(N+k)}{k ! \Gamma(N)}\left|1-t_{j}\right|^{k}\right\}^{q}\|u\|_{H^{q}}^{q} .
$$

The boundedness of $u C_{\varphi}$ implies that $u \in H^{q}\left(B_{N}\right)$ and the convergence of the series $\sum(\Gamma(N+$ $k) / k ! \Gamma(N))\left|1-t_{j}\right|^{k}$ implies that

$$
\sum_{k=n}^{\infty} \frac{\Gamma(N+k)}{k ! \Gamma(N)}\left|1-t_{j}\right|^{k} \longrightarrow 0 \quad \text { as } n \longrightarrow \infty
$$

So we obtain

$$
\int_{\left(1-t_{j}\right) \overline{B_{N}}}\left|R_{n} f\right|^{q} d \mu_{u, \varphi} \longrightarrow 0 \quad \text { as } n \longrightarrow \infty
$$

Combining (3.27), (3.28), and (3.31) with Lemma 3.6, we have

$$
\left\|u C_{\varphi}\right\|_{e, H^{p} \rightarrow H^{q}}^{q} \leq \liminf _{n \rightarrow \infty}\left\|u C_{\varphi} R_{n}\right\|_{H^{p} \rightarrow H^{q}}^{q} \leq C \sup _{n \geq 1}\left\|R_{n}\right\|^{q}\left(M_{j}+\varepsilon\right) .
$$

Since Corollary 3.4 implies that $\sup _{n \geq 1}\left\|R_{n}\right\|<\infty$, and $\varepsilon>0$ was arbitrary, we conclude that

$$
\left\|u C_{\varphi}\right\|_{e, H^{p} \rightarrow H^{q}}^{q} \leq\left\{\begin{array}{l}
C \limsup _{|w| \rightarrow 1^{-}} \int_{\partial B_{N}}\left|u^{*}(\zeta)\right|^{q}\left\{\frac{1-|w|^{2}}{\left|1-\left\langle\varphi^{*}(\zeta), w\right\rangle\right|^{2}}\right\}^{q N / p} d \sigma(\zeta), \\
C \limsup _{t \rightarrow 0^{+}} \sup _{\zeta \in \partial B_{N}} \frac{\mu_{\varphi, u}(S(\zeta, t))}{t^{q N / p}}
\end{array}\right.
$$

which were to be proved.

Corollary 3.7 (see [15]). Suppose that $1<p \leq q<\infty$. For the bounded weighted composition operator $u C_{\varphi}: H^{p}\left(B_{N}\right) \rightarrow H^{q}\left(B_{N}\right)$, the following conditions are equivalent:

(a) $u C_{\varphi}: H^{p}\left(B_{N}\right) \rightarrow H^{q}\left(B_{N}\right)$ is compact;

(b) $u$ and $\varphi$ satisfy

$$
\lim _{|w| \rightarrow 1^{-}} \int_{\partial B_{N}}\left|u^{*}(\zeta)\right|^{q}\left\{\frac{1-|w|^{2}}{\left|1-\left\langle\varphi^{*}(\zeta), w\right\rangle\right|^{2}}\right\}^{q N / p} d \sigma(\zeta)=0 ;
$$

(c) $u$ and $\varphi$ satisfy

$$
\lim _{t \rightarrow 0^{+}} \sup _{\zeta \in \partial B_{N}} \frac{\mu_{\varphi, u}(S(\zeta, t))}{t^{q N / p}}=0
$$




\section{Multiplication operators between Hardy spaces}

In this section, we consider the compact multiplication operator $M_{u}$ between Hardy spaces. As a consequence of Theorem 3.1, we obtain the following results.

Corollary 4.1. Suppose that $1<p \leq q<\infty$. For the bounded multiplication operator $M_{u}: H^{p}\left(B_{N}\right) \rightarrow$ $H^{q}\left(B_{N}\right)$, the following inequality holds:

$$
\left\|M_{u}\right\|_{e, H^{p} \rightarrow H^{q}}^{q} \sim \limsup _{|w| \rightarrow 1^{-}} \int_{\partial B_{N}}\left|u^{*}(\zeta)\right|^{q}\left\{\frac{1-|w|^{2}}{|1-\langle\zeta, w\rangle|^{2}}\right\}^{q N / p} d \sigma(\zeta) .
$$

Furthermore, $M_{u}: H^{p}\left(B_{N}\right) \rightarrow H^{q}\left(B_{N}\right)$ is compact if and only if

$$
\lim _{|w| \rightarrow 1^{-}} \int_{\partial B_{N}}\left|u^{*}(\zeta)\right|^{q}\left\{\frac{1-|w|^{2}}{|1-\langle\zeta, w\rangle|^{2}}\right\}^{q N / p} d \sigma(\zeta)=0 .
$$

By using Corollary 4.1, we can completely characterize the compactness of a multiplication operator $M_{u}$ from $H^{p}\left(B_{N}\right)$ into $H^{q}\left(B_{N}\right)$.

Theorem 4.2. Suppose that $1<p \leq q<\infty$. Then $M_{u}: H^{p}\left(B_{N}\right) \rightarrow H^{q}\left(B_{N}\right)$ is compact if and only if $u=0$ in $B_{N}$.

Proof. If $u \equiv 0$, then $M_{u}$ is compact. Thus, we only prove that the compactness of $M_{u}$ implies $u \equiv 0$. The boundedness of $M_{u}$ implies that $u \in H^{q}\left(B_{N}\right)$. Hence, the Poisson representation for $u$ gives that

$$
u(w)=\int_{\partial B_{N}} u^{*}(\zeta) P(w, \zeta) d \sigma(\zeta) \quad\left(w \in B_{N}\right)
$$

where $P(w, \zeta)$ is the Poisson kernel. Hölder's inequality shows that

$$
\begin{aligned}
|u(w)| & \leq \int_{\partial B_{N}}\left|u^{*}(\zeta)\right| P(w, \zeta) d \sigma(\zeta) \\
& \leq\left\{\int_{\partial B_{N}}\left|u^{*}(\zeta)\right|^{q} P(w, \zeta)^{q / p} d \sigma(\zeta)\right\}^{1 / q}\left\{\int_{\partial B_{N}} P(w, \zeta)^{(1-1 / p) q^{\prime}} d \sigma(\zeta)\right\}^{1 / q^{\prime}},
\end{aligned}
$$

where $1 / q+1 / q^{\prime}=1$. By the assumption $1<p \leq q<\infty$, we see that

$$
s \equiv\left(1-\frac{1}{p}\right) q^{\prime}=\frac{q(p-1)}{p(q-1)} \leq \frac{p q-p}{p(q-1)}=1,
$$

and so we have

$$
\int_{\partial B_{N}} P(w, \zeta)^{(1-1 / p) q^{\prime}} d \sigma(\zeta) \leq\left\{\int_{\partial B_{N}}\left\{P(w, \zeta)^{s}\right\}^{1 / s} d \sigma(\zeta)\right\}^{s}=1 .
$$


Inequality (4.4) and Corollary 4.1 give that $\lim _{|w| \rightarrow 1^{-}}|u(w)|=0$. Since $u \in H^{q}\left(B_{N}\right)$, this implies that $u$ has a $K$-limit 0 on a set of positive $\sigma$-measure in $\partial B_{N}$. Hence [17, page 83, Theorem 5.5.9] shows that $u \equiv 0$. This completes the proof.

\section{Acknowledgment}

The authors would like to thank the referee for the careful reading of the first version of this paper and for the several suggestions made for improvement.

\section{References}

[1] B. R. Choe, "The essential norms of composition operators," Glasgow Mathematical Journal, vol. 34, no. 2, pp. 143-155, 1992.

[2] P. Gorkin and B. D. MacCluer, "Essential norms of composition operators," Integral Equations and Operator Theory, vol. 48, no. 1, pp. 27-40, 2004.

[3] P. Poggi-Corradini, "The essential norm of composition operators revisited," in Studies on Composition Operators, vol. 213 of Contemporary Mathematics, pp. 167-173, American Mathematical Society, Providence, RI, USA, 1998.

[4] J. H. Shapiro, "The essential norm of a composition operator," Annals of Mathematics, vol. 125, no. 2, pp. 375-404, 1987.

[5] S. Li and S. Stević, "Weighted composition operators between $H^{\infty}$ and $\alpha$-Bloch space in the unit ball," to appear in Taiwanese Journal of Mathematics.

[6] S. Li and S. Stević, "Weighted composition operators from $H^{\infty}$ to the Bloch space on the polydisc," Abstract and Applied Analysis, vol. 2007, Article ID 48478, 13 pages, 2007.

[7] G. Mirzakarimi and K. Seddighi, "Weighted composition operators on Bergman and Dirichlet spaces," Georgian Mathematical Journal, vol. 4, no. 4, pp. 373-383, 1997.

[8] S. Ohno, "Weighted composition operators between $H^{\infty}$ and the Bloch space," Taiwanese Journal of Mathematics, vol. 5, no. 3, pp. 555-563, 2001.

[9] S. Ohno, K. Stroethoff, and R. Zhao, "Weighted composition operators between Bloch-type spaces," The Rocky Mountain Journal of Mathematics, vol. 33, no. 1, pp. 191-215, 2003.

[10] S. Ohno and R. Zhao, "Weighted composition operators on the Bloch space," Bulletin of the Australian Mathematical Society, vol. 63, no. 2, pp. 177-185, 2001.

[11] M. D. Contreras and A. G. Hernández-Díaz, "Weighted composition operators on Hardy spaces," Journal of Mathematical Analysis and Applications, vol. 263, no. 1, pp. 224-233, 2001.

[12] M. D. Contreras and A. G. Hernández-Díaz, "Weighted composition operators between different Hardy spaces," Integral Equations and Operator Theory, vol. 46, no. 2, pp. 165-188, 2003.

[13] Z̆. Čučković and R. Zhao, "Weighted composition operators on the Bergman space," Journal of the London Mathematical Society, vol. 70, no. 2, pp. 499-511, 2004.

[14] Z̆. Čučković and R. Zhao, "Weighted composition operators between different weighted Bergman spaces and different Hardy spaces," Illinois Journal of Mathematics, vol. 51, no. 2, pp. 479-498, 2007.

[15] S. Ueki, "Weighted composition operators between weighted Bergman spaces in the unit ball of $\mathbb{C}^{n}$," Nihonkai Mathematical Journal, vol. 16, no. 1, pp. 31-48, 2005.

[16] L. Luo, "The essential norm of a composition operator on Hardy space of the unit ball," Chinese Annals of Mathematics (Series A, Chinese), vol. 28A, no. 6, pp. 805-810, 2007, preprint.

[17] W. Rudin, Function Theory in the Unit Ball of $\mathbb{C}^{n}$, vol. 241 of Fundamental Principles of Mathematical Science, Springer, New York, NY, USA, 1980.

[18] S. C. Power, "Hörmander's Carleson theorem for the ball," Glasgow Mathematical Journal, vol. 26, no. 1, pp. 13-17, 1985.

[19] B. D. MacCluer, "Compact composition operators on $H^{p}\left(B_{N}\right)$," The Michigan Mathematical Journal, vol. 32, no. 2, pp. 237-248, 1985.

[20] K. H. Zhu, "Duality of Bloch spaces and norm convergence of Taylor series," The Michigan Mathematical Journal, vol. 38, no. 1, pp. 89-101, 1991.

[21] C. C. Cowen and B. D. MacCluer, Composition Operators on Spaces of Analytic Functions, Studies in Advanced Mathematics, CRC Press, Boca Raton, Fla, USA, 1995. 


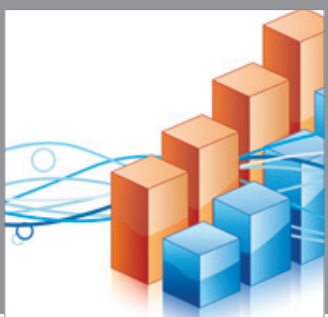

Advances in

Operations Research

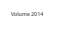

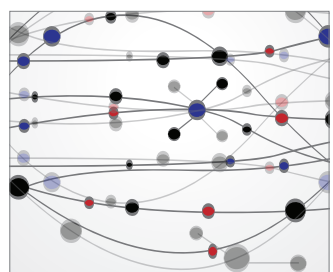

\section{The Scientific} World Journal
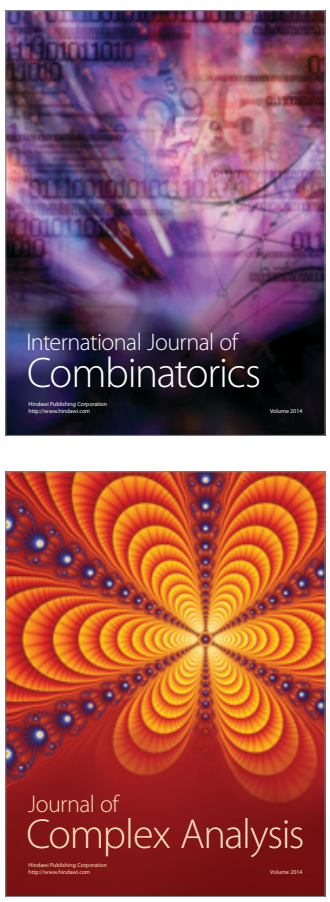

International Journal of

Mathematics and

Mathematical

Sciences
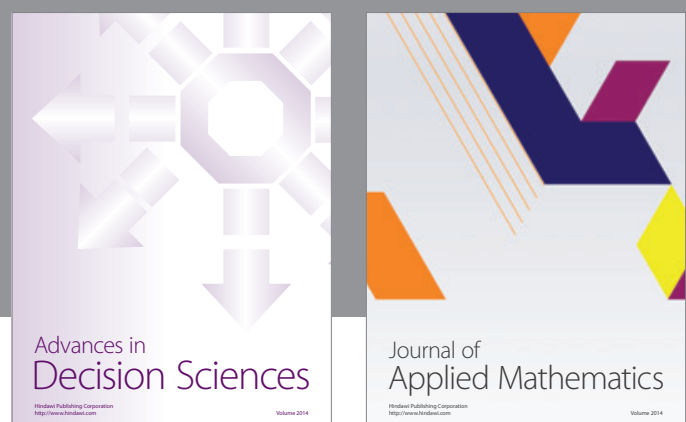

Journal of

Applied Mathematics
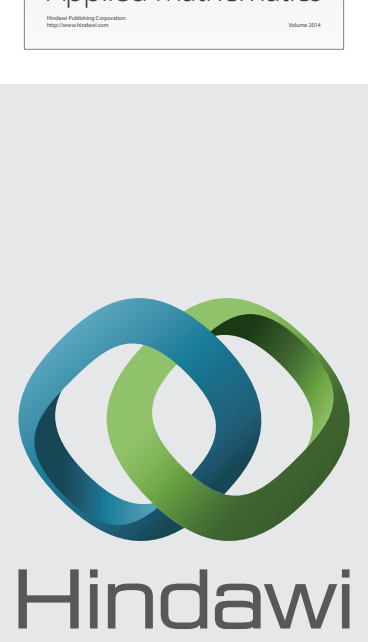

Submit your manuscripts at http://www.hindawi.com
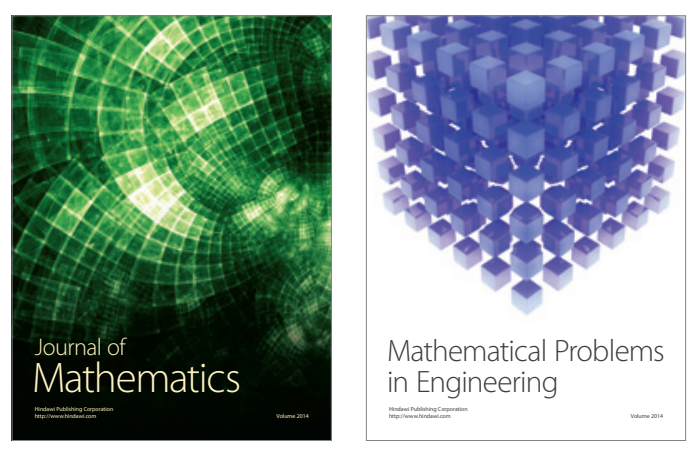

Mathematical Problems in Engineering
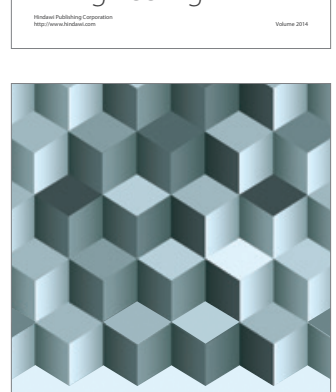

Journal of

Function Spaces
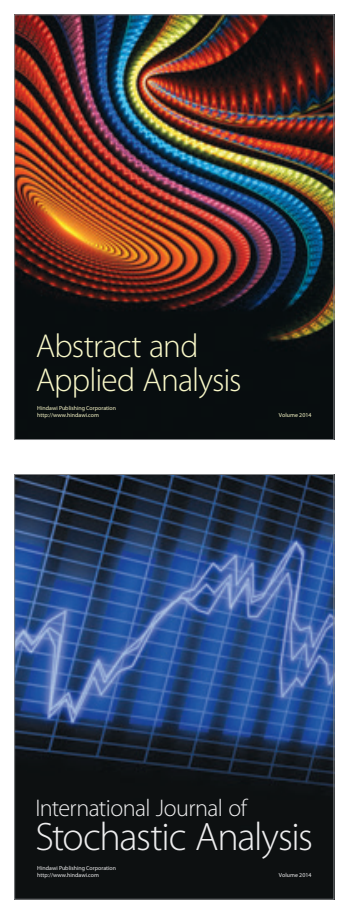

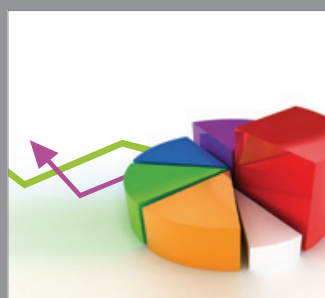

ournal of

Probability and Statistics

Promensencen
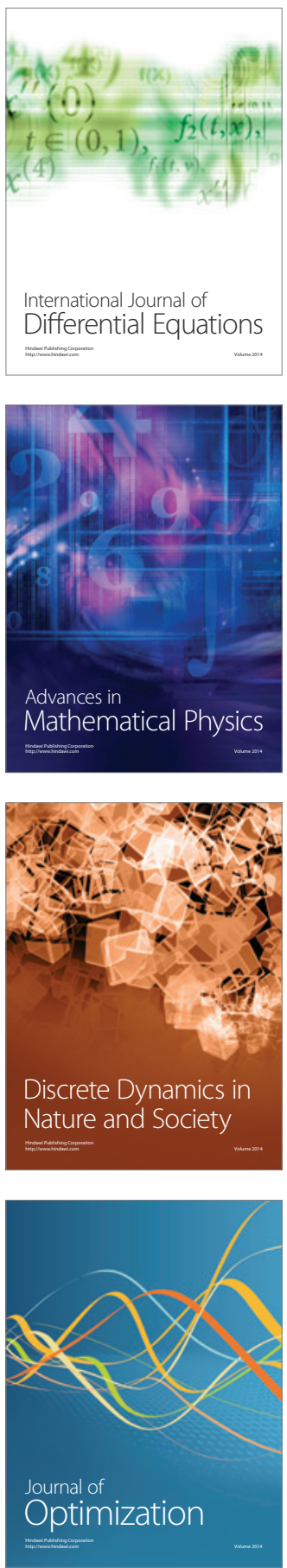